\title{
ON THE CAUSE OF THE RESPIRATORY VARIATION OF THE BALLISTOCARDIOGRAM, WITH A NOTE ON SINUS ARRHYTHMIA
}

\author{
BY ISAAC STARR AND CARL K. FRIEDLAND \\ (From the Research Department of Therapeutics and the Medical Clinic of the Hospital of the \\ University of Pennsylvania, Philadelphia)
}

(Received for publication July 5, 1945)

Variation in the size of the impacts during the respiratory cycle is a feature of almost every ballistocardiogram (1). As the subject inhales, the impacts increase in size, to diminish again as he exhales; if the breath is held, this rhythmic variation disappears. The purpose of this investigation is to study the causes of this respiratory variation.

At first glance the interpretation seemed easy. The old physiological viewpoint that inspiration sucks blood as well as air into the chest, and so causes both an increased cardiac filling and a corresponding increase in cardiac output, seemed a sufficient explanation of our findings, especially since the results of animal experiments (2) were concordant with this view. But, several facts made us hesitate to adopt it unreservedly without further study.

The first difficulty was concerned with the interpretation of the results of experiments on the peripheral circulation performed by several workers (3 to 5 ). These studies demonstrated a respiratory variation in volume and volume pulse of the digits which seemed out of phase with, and so not to be attributed to, the respiratory changes in the cardiac output which one might, at first thought, expect from the ballistocardiogram.

A second discrepancy was in the behavior of the usual respiratory variation of the arterial blood pressure (6). Using the sphygmomanometer, we convinced ourselves that the peaks of blood pressure did not coincide with the maximum impacts of the respiratory cycle, a gross observation confirmed by the results of more refined methods recorded in this paper.

Another observation seemed at first difficult to explain. If the glottis is kept open when the breath is held after a deep inspiration, the ballistic impacts continue large; when held after a deep expiration, the impacts remain small until respiration is resumed. This finding, added to those mentioned previously, made us stop to consider an alternative explanation of the ballistocardiogram's respiratory variation as follows.

The ballistocardiograph, as it is usually employed, records the resultant of the force vectors in the long axis of the body. As the diaphragm rises during expiration, the cardiac apex moves to the left rotating the heart's axis counter-clockwise. Such a movement would diminish the recorded force because the vectors, due to movement of blood in the heart, would be thrown out of the line in which we are recording. Such a mechanism might explain the common respiratory variations of the ballistocardiogram without invoking any changes in cardiac output at all, and it gives a simple explanation why, when the breath is held at full inspiration or expiration, the impacts continue large or small until the diaphragm returns to its usual place; it might be entirely a matter of the heart's position.

Ordinarily, the respiratory movements alter the position of the heart and also change the effective pressure gradient in the blood which fills it. It was our plan to conduct experiments to separate the effects due to changes of the heart's position from those caused by changes in the filling pressure, and so learn the major cause of the respiratory variations in the impacts.

In addition, we unexpectedly encountered a clinical situation which permitted a qualitative study of the effects of respiratory movements on the output from each side of the heart separately. Stimulated by the ideas suggested by these results, we sought further information by simultaneous records of blood pressure and the ballistocardiogram. The conclusions to be drawn from our results agree with a well-known physiological viewpoint recently supported by results obtained in animal experiments (7). Pressure changes, and not changes in position of the heart, cause the respiratory variations of the ballistocardiogram. 
Also, our experiments have provided us with a new insight into the genesis of the common respiratory arrhythmia of young persons, the so-called sinus arrhythmia.

The first of the experiments to be reported was performed early in the studies on the ballistocardiograph by the senior author, and it was repeated in 1944. Opportunity for the second study came in 1943. ${ }^{1}$ The remainder of the work was completed in 1945 by both of the authors together.

\section{EXPERIMENTS}

These fall into 4 groups, and the technique and results can best be described separately.

1. Attempts to reverse the respiratory variation by changing the subject's position on the ballistocardiograph.

Let us assume that expiration diminishes the ballistocardiagram's excursion because the change in the heart's position moves the force vectors out of the line in which we usually record them. Then if one changes the position of the subject on the ballistocardiograph until its long axis is in line with the heart's axis at expiration, the normal effect of respiration on the ballistocardio-

1 This study was made through the kindness of Dr. R. H. Cheney. gram should be reversed, because expiration now brings the vectors into the line in which we are recording.

Therefore, after determining the heart's axis at full expiration by the $\mathrm{x}$-ray, we placed a normal subject on the ballistocardiograph in such a position that this axis coincided as nearly as possible with the long axis of that instrument. To be more certain of securing a picture with the subject in exactly the right position, a number of ballistocardiograms were taken with the subject at slightly different angles on the table and with different degrees of rotation of his body about its long axis. In two normal subjects, we were unable to find any position in which the effect of respiration on the ballistocardiogram was reversed.

2. Experiments on a subject in the decerebrate condition.

If there were no muscular movement to distort it, artificial respiration by blasts of air would reverse the normal relation between pressure in the lungs and the position of the heart. Thus, inspiration induced by a blast would drive the diaphragm down and bring the heart toward the midline as in normal inspiration, but the intrapulmonary and the intrathoracic pressures, instead of being reduced during inspiration, would be increased.

Fig. 1. Respiratory Effects on the Ballistocardiogram

The reproductions are 50 per cent of actual size. The time records shown apply to all records; the longest interval is one second. The white line above represents position of chest and moves upward during inspiration. The black line is pressure in the mask. The calibration was essentially linear and is noted for each record; the larger mark is placed at atmospheric pressure and the second mark at $20 \mathrm{~cm} . \mathrm{H}_{2} \mathrm{O}$ above this. The correction for parallax is negligible. Slanting lines point to features of interest.

First record (top): Subject C. F., age 30, height $5 \mathrm{ft}$. $10 \%$ in., weight 175 pounds. A normal subject. Normal breathing is represented. The mask pressure record hardly shows on the reproduction, and its fluctuations are very small. Note sinus arrhythmia and ballistic complexes increasing in size during inspiration.

Second record: C. F., during periodic inflation of lungs with positive pressure. Note reversal of respiratory effects on the ballistocardiogram, the ballistic complexes decreasing in size during inspiration. A typical respiratory impact is marked.

Third record: C. F., during periodic inflation at higher pressure. Note the general reduction in size of complexes when compared with the top record. Complexes are smallest during inspiration. Note two complexes of abnormal form, doubled " $\mathrm{J}$ " peaks, occurring just after initial rise of pressure. These are marked by oblique lines.

Fourth record (left) : Subject I. S., age 50, height $6 \mathrm{ft}$., weight 185 pounds. A normal subject. The subject voluntarily breathed deeply to bring out the normal respiratory variations. Note absence of sinus arrhythmia.

Fourth record (right) : Subject H. H., age 20, height $5 \mathrm{ft}$., weight 140 pounds, B. P. $90 / 70 \mathrm{~mm}$. Hg. Subject in the decerebrate condition following an unsuccessful attempt to remove a brain tumor. No spontaneous respiration; at the mark the lungs were inflated by a blast of oxygen down a tracheal catheter. Note diminished ballistic amplitude when lungs are expanded by the positive pressure, a reversal of the usual respiratory effect.

Fifth record: Subject I. S., under positive pressure inflation. Note the generally smaller complexes and the appearance of a respiratory arrhythmia, the smaller complexes occurring at a slower rate. Note that the size of the impacts is maintained for a few beats after each increase of pressure; after this, they diminish in amplitude until pressure approaches zero.

Sixth record (left) : F. B., age 60 , height $5 \mathrm{ft}$., weight 


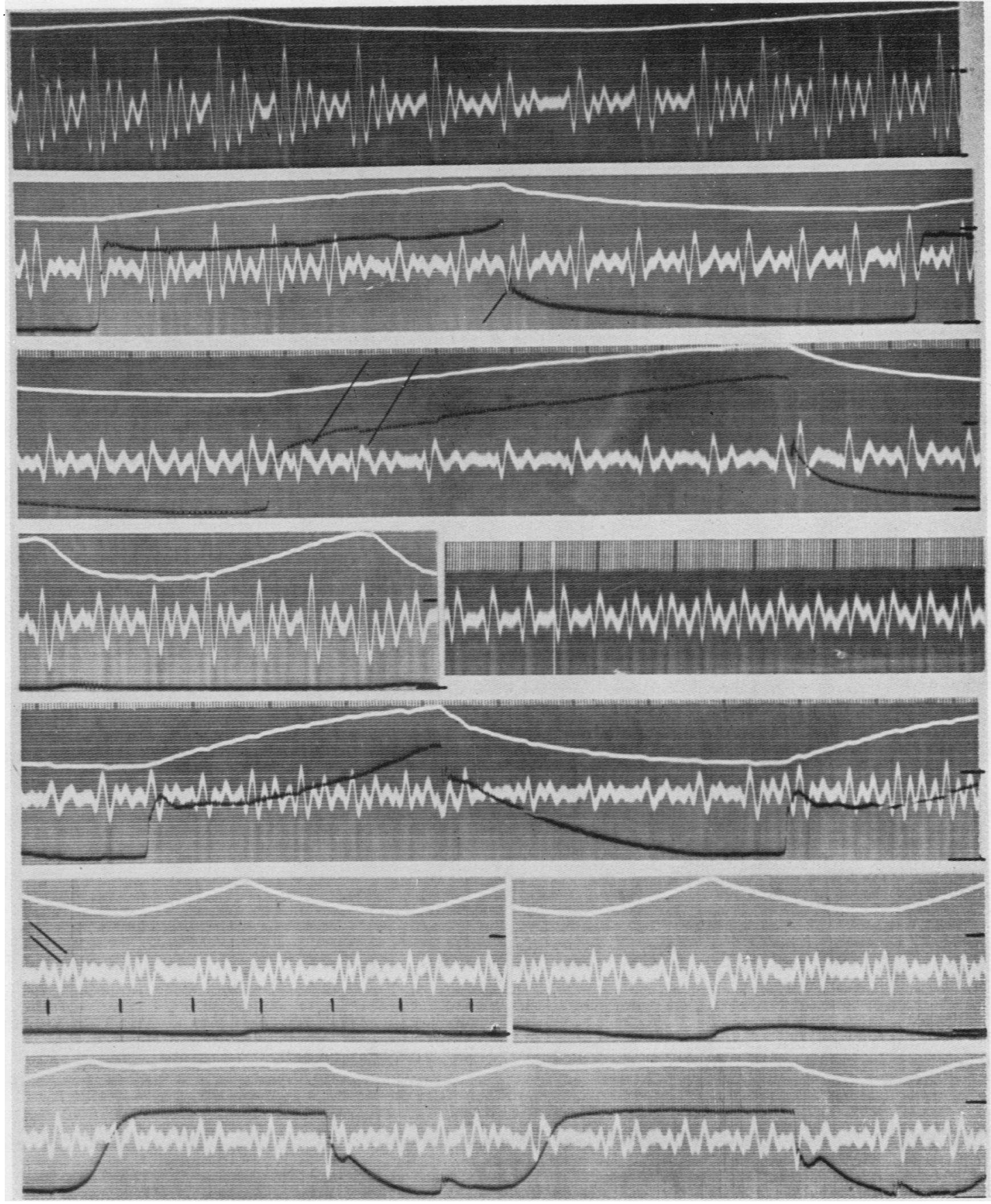

FIG. 1.

145 pounds. A patient with an aneurism of the left ventricle. Vertical marks below the ballistocardiogram show the location of the beginning of the QRS of lead 1 of an electrocardiogram taken simultaneously. Note that the position of electrical systole demonstrates that the upward deflection immediately following it is the " $\mathrm{H}$ " wave, although it is the largest upward deflection seen in many complexes. The "J" wave has 2 peaks, and these are marked in the first complex. Note that the height of each "J" peak varies with the phases of respiration. Note the downward respiratory impact when the diaphragm abruptly changes its direction at full inspiration.

Sixth record (right) : F. B., during voluntary increased breathing.

Seventh record: F. B. during periodic positive pressure inflation. Note that during periods of high pressure, the second " $\mathrm{J}$ " peak is higher than the first. When pressure is low, the reverse is true. 
After craniotomy, in an attempt to remove a brain tumor which proved to be inoperable, patient $\mathrm{H}$. H. went into the decerebrate condition. At the time of our tests, she was unconscious without spontaneous respiratory movements but with a reasonably normal circulation, the blood pressure being 90/70, the pulse rate about 120 . Life was maintained by artificial respiration with oxygen blown through a catheter inserted to the tracheal bifurcation.

Figure 1 shows the ballistocardiogram when the lungs were inflated by a blast of oxygen. The increased pressure distended the chest, but it diminished the size of the "I" and "J" waves; therefore, the normal respiratory variation was reversed. This was repeated many times with the same result.

Attempts to restore active respiration were unsuccessful, and the patient eventually died about 12 hours after the observations mentioned above.

3. Experiments on a subject aith ventricular aneurism.

In an early experiment, when the right ventricular wall of a dog had been damaged until it bulged with each systole, a change in the form of the ballistocardiogram had occurred, the " $J$ " wave changing from the normal single peak to a doubled peak (1). Therefore, we were not surprised to find that the ballistocardiogram of a patient with a cardiac aneurism also showed a doubled "J" peak.

F. B., a man of 60.5 feet tall, 145 pounds in weight. came to the hospital with a history of slight gastro-intestinal bleeding. He had had an episode of substernal pain 3 years before but belittled it and denied all symptoms referable to the cardiovascular system. Blood pressure was $108 / 62$. The cardiac silhouette showed a pronounced bulge of the left ventricular wall, and fluoroscopy ${ }^{2}$ demonstrated an aneurism the size of half a tennis ball on the anterior superior aspect of the left ventricle. This aneurism expanded outward about $1 \mathrm{~cm}$. at each systole as the remainder of the wall contracted. The heart was not otherwise enlarged. Electrocardiogram suggested an old infarction of the anterior portion of the left ventricle, perhaps involving the septum. A gastric lesion was demonstrated by $\mathrm{x}$-ray and,

2 This was performed by Dr. A. Margolies. as carcinoma was suspected, laparotomy was thought necessary. A chronic peptic ulcer was found and a partial gastrectomy performed. $\mathrm{He}$ stood the operation well, and had an uneventful convalescence. Ballistocardiograms taken before and after operation were entirely similar.

This patient's ballistocardiograms (Figure 1) were always grossly abnormal in form, the " $\mathrm{J}$ " peak being replaced by two small peaks, causing an abnormality of the late "M" type. Since this type of abnormality had been expected in cardiac aneurism from theoretical conceptions, as well as produced experimentally (1), it seems reasonable to suppose that the first " J" peak, occurring at the normal time, was due to impacts from the more normal right heart; whereas, the delayed second peak was to be attributed to the abnormal left heart. The force of its contractions must have distended the aneurism before sufficient pressure was raised to permit rapid ejection into the aorta and, consequently, acceleration of the blood would be both retarded and diminished, and the resulting impacts both late and small.

The fact that data obtained in this case would contribute to our study of the ballistic respiratory variation was made manifest by the observation that the size of both peaks of the " $J$ " waves varied with respiration, and that the two variations were not synchronous.

To study these independent variations, the respiratory cycle was divided into 4 parts as shown in Figure 2. The height of all "J" waves on our records was measured, and the data divided into 4 groups corresponding to the part of the respiratory cycle in which the impacts fell. The averages of each group, plotted in Figure 2 in the middle of that part of the respiratory cycle in which they occurred, show how the 2 peaks vary in size with this cycle. There was, however, much uncertainty about the value to be given to the second peak $\left(\mathrm{J}_{2}\right)$ at the height of inspiration, at which time it was little more than a shoulder on the descending limb of $\mathrm{J}_{1}$. Therefore a question mark has been placed at this position in Figure 2.

A record of the same patient during artificial positive pressure respiration by the technique described below is shown in Figure 1. After the pressure had been raised, the first " $J$ " peak was smaller than the second; when the pressure had 
fallen, the reverse was the case in all complexes free of distortion by diaphragmatic impacts.

Interest in the interpretation of the respiratory changes in this patient led us to make a series of more elaborate experiments on normal persons.

\section{SUBJECT F.B. L. VENTRIC. ANEURISM}

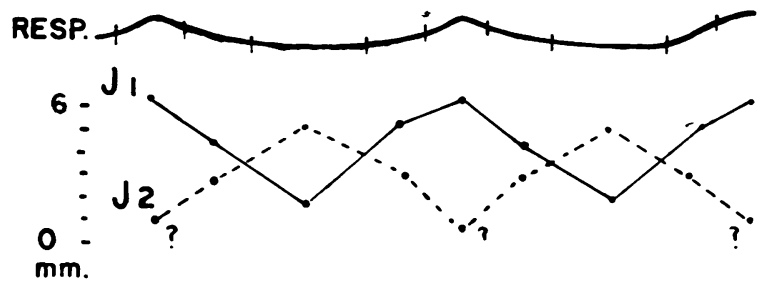

Fig. 2. Independent Respiratory Variation of the Two "J" Waves of the Ballistocardiogran in a Case of Ventricular Axeurisy

The respiratory cycle shown at the top has been divided into four parts as shown, the line moving upward on inspiration. The values given below are average heights of the two " $\mathrm{J}$ " waves occurring in that part of the respiratory cycle, measured from the record's base line.

\section{Experinents on normal subjects.}

The 4 normal subjects used were all males being 24, 25, 30, and 50 years of age. A patient, aged 61 years, also served as a subject and gave concordant results. All the measurements described below were not made on every subject; blood pressure was recorded continuously in only 3 of the 5 , but positive pressure was given to all.

A half-face mask with a pneumatic cushion was employed. It had been altered by the introduction of a sccond opening to permit insertion of a tube leading to a capsule with rubber membrane and mirror for the measurement of pressure in the mask. This mask was held fast by straps around the head.

Positive pressures were secured from a tank of compressed air with a reducing valve. A device for artificial respiration, the Pneumolator, made by the General Electric Co., was used to provide intermittent interruption of the air blast.

To record the position of the chest wall, a rubber bag was bandaged around the lower rib margins and connected, in early experiments, with a tambour and lever which interrupted the light beam. In later experiments, a capsule, with a tight rubber membrane and a mirror, was employed.
An 18-gauge needle, connected by lead tubing to a Hamilton manometer $(8),{ }^{3}$ was inserted into the femoral artery after anesthetization by procaine. The natural frequency of the manometer system was approximately 150 per second. The light beams from all the recording devices were thrown into the ballistocardiograph's camera, and so all the records were made simultaneously.

The subject lay at rest on the horizontal ballistocardiograph for $1 \%$ hour or longer before any observations were made. Records were then taken during normal breathing and during voluntary deep and slow breathing. After this, the mask's air inlet was obstructed by a cork containing a hole $7 \mathrm{~mm}$. or less in diameter, and records were made both during natural breathing and voluntary deep breathing through this obstruction.

Finally the mask was connected through the pneumolator to a tank of compressed air and positive pressure applied intermittently. We aimed to adjust the blasts so that the rhythm was slow and the pressure curves as flat as possible, but we were not always able to accomplish this. The subjects were instructed to relax completely and let the apparatus breathe for them, but the tendency to make respiratory movements was strong, and the results obtained could not be completely controlled by changing the adjustment of the blast. So the pressure curves obtained varied greatly as will be seen in the figures.

Tests for parallax and calibration of the capsules and manometer completed the experiment.

The results obtained on all 5 subjects were entirely concordant. Typical results are shown in Figures 1 and 3 . In interpreting these records, one must carefully distinguish between impacts due to movement of blood and those due to movement of the organs whose position changes with respiration. When the diaphragm rapidly changes its direction, especially at the height of inspiration, an impact is often recorded by the ballistocardiograph. Instances of this will be found in many records, especially when positive pressure was employed. Occurring simultaneously with rapid changes in pressure, or of position of the chest, these respiratory impacts can readily be identified.

\footnotetext{
${ }^{3}$ We are indebted to Dr. C. C. Wolferth, of the Robinette Foundation for the loan of essential parts of this instrument.
} 


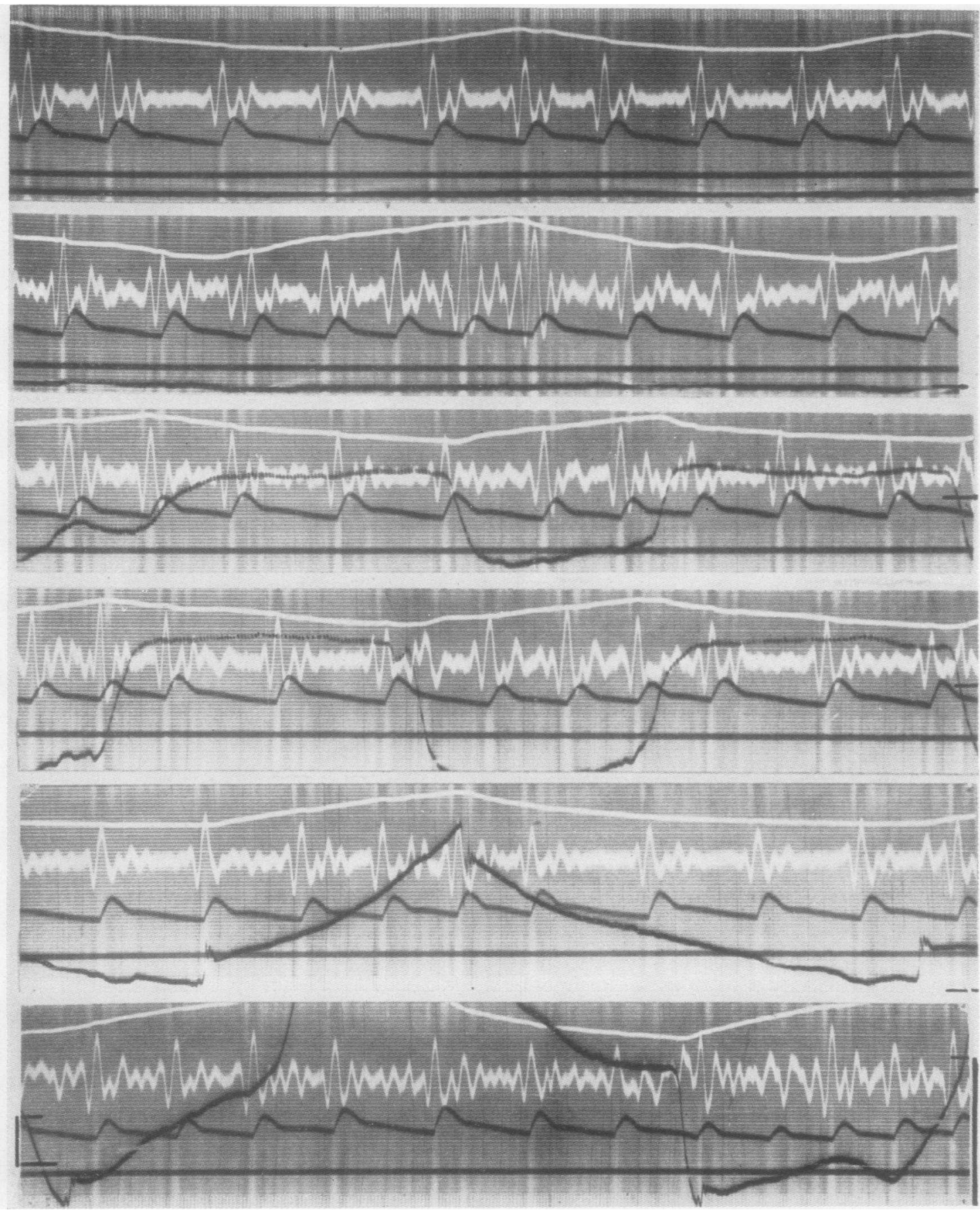

Fig. 3. Respiratory Effects on Ballistocardiogram and Arterial Blood Pressure

The time records shown apply to all records: the longest interval is one second. The white line, below the time in the top record, shows position of the chest; it moves upward during inspiration. The white line next below is the ballistocardiogram. The black line below it is the record of the Hamilton manometer, the stationary black line is the blood pressure reference line; note that it is not exactly at zero. The next black line, the lowest in the top record is mask pressure; in the lower four records this line crosses the others.

Calibration showed both scales to be almost linear. B.P. scale is given to the left of the lowest tracing, the lower mark is at zero; i.c., at atmospheric pressure; the upper has been placed at $100 \mathrm{~mm}$. $\mathrm{Hg}$. This calibration applies 
Features of our results important to our argument are pointed out in the legends of Figures 1 and 3 , and their meaning will be discussed in the text.

\section{DISCUSSION}

All our evidence shows that change of pressure, rather than of the heart's position, causes the respiratory variation seen in the normal ballistocardiogram. Changing the position of the subject, until we record in the axis of the heart at expiration, fails to reverse the respiratory variation. Breathing through an obstruction, and thus exaggerating the pressure differences of the cycle without influencing the changes of the heart's position, greatly increases the respiratory variation of the impacts. Artificial respiration with a blast of air, which reverses the usual relation between pulmonary pressures and the heart's position, profoundly changes the respiratory variation, sometimes reversing it completely as shown in Figure 1, record 2 , although at other times, such as the last record in Figure 3, the effect is not so strikingly different from that seen in normal breathing, for reasons to be discussed below.

According to our theoretical conceptions, displacement of the heart by a rising diaphragm would have less effect on the recorded impacts than one might suppose. There are several reasons for this; first, a large part of the recorded impact comes from the movement of blood in the aorta, an organ whose position remains fixed dur-

to all records, as does the relation between the blood pressure reference line and zero pressure.

The mask pressure scale is given to the right of the lowest record. The bottom of this record is at zero; i.e., at atmospheric pressure, the upper mark $20 \mathrm{~cm} . \mathrm{H}_{2} \mathrm{O}$ above this level. This calibration applies to all records. The point of zero (atmospheric) pressure is marked on each record.

All records were obtained on the same healthy subject. S. H., age 24, weight 145 pounds, height $5 \mathrm{ft}$., $91 / 2$ in.

Top record: Normal breathing, note smallness of respiratory variations of ballistocardiogram and blood pressure.

Second record: Voluntary increased breathing. Note increased respiratory effect on ballistocardiogram and B.P.; during inspiration, the ballistocardiogram increases in amplitude, systolic pressure falls $15 \mathrm{~mm} . \mathrm{Hg}$, and pulse pressure diminishes from 55 to $45 \mathrm{~mm}$. $\mathrm{Hg}$.

Third record: Normal breathing with the air-way partly obstructed. B.P. falls from $118 / 60$ to $102 / 57$ on inspiration, while ballistocardiogram is increasing in amplitude. ing the respiratory cycle; second, the axes of the right and left hearts are not parallel and the small pendulum movements of respiration when throwing one axis out would bring the other more into line ; third, while displacement of the heart diminishes our record of one force vector, it brings new and partly compensating vectors into being.

The principal elements concerned can be visualized by reference to Figure 4 . The presentation is not strictly quantitative as the vectors are not given a dimension in time, nor can they be accurately placed in time, but we believe that it will be more easily understood by most readers for that reason. The strict mathematical treatment of ballistocardiographic theory has been presented (9), and this treatment could easily be applied to the present problem by any one interested. It depends on assumptions of the nature of the ejection velocity curve, and indeed of other factors about which little is known (19). Therefore, we have contented ourselves with a simple and not strictly quantitative presentation in this instance.

We expect, therefore, that small deviations of the cardiac axis like those occurring during the respiratory cycle would not produce a noteworthy effect on the record; and that a large shift of the heart's position, such as sometimes occurs after the operative removal of one lung,. would give a record with an "I" wave diminished out of proportion to the rest of the systolic complex. This abnormality of form has indeed been found in such a case (9), and the results in this paper are

Fourth record: Voluntary increased breathing with the air way partly obstructed.

Fifth record: Positive pressure inflation. Complexes as a whole are reduced in size. Note that compared with record 2, in which chest expansion is comparable, there is much less increase in the impacts during inspiration. Also, under positive pressure, the complexes increase somewhat in size during expiration, while in record 2 they diminish markedly.

Sixth record: Higher positive pressure inflation. The mask pressure record ran off the camera film but was observed on the scale at the camera aperture. The maximum mask pressure reached $37 \mathrm{~cm} . \mathrm{H}_{2} \mathrm{O}$. Note lack of the usual increase of ballistocardiogram amplitude during inspiration. Indeed, the impacts become smaller as long as mask pressure remains above atmospheric pressure. Note direct relation between mask pressure and blood pressure. The changes in systolic pressure can be accounted for by the changes in mask pressure. Note production of marked "paradoxical pulse." 


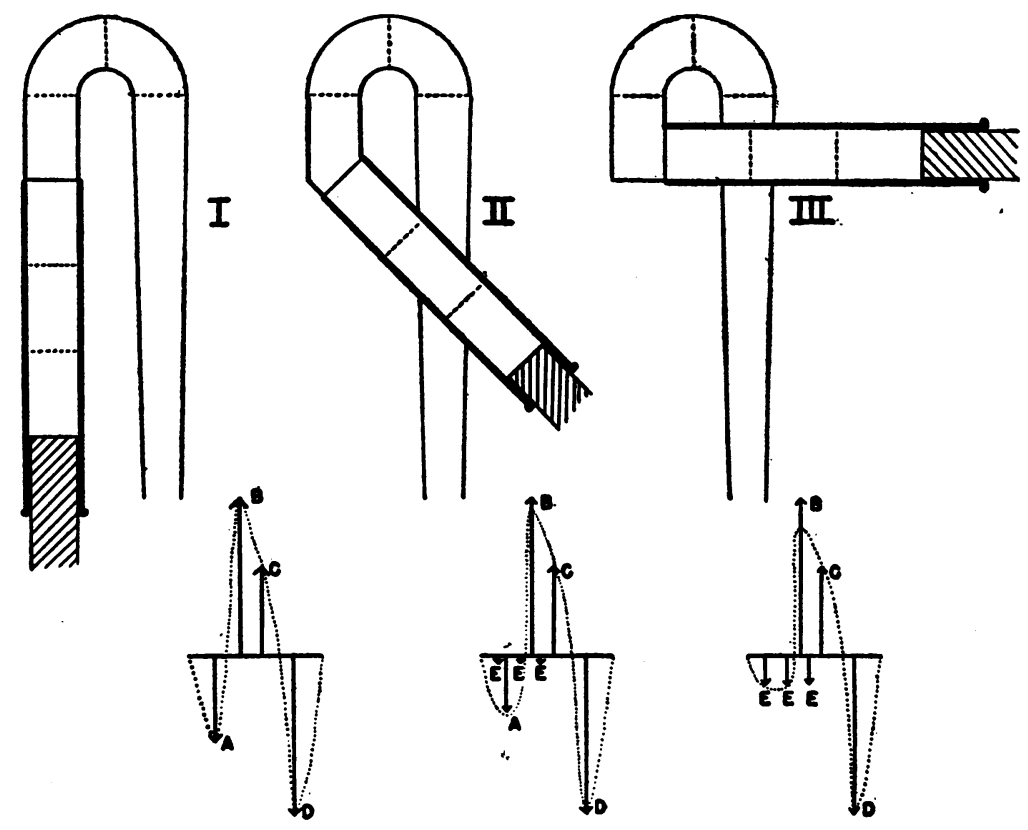

Fig. 4. Schematic Drawing to Illustrate the Theoretical Effect of Cardiac Displacement on THE BALLISTOCARDIOGRAM

A piston syringe is connected to an "aorta." In I, the syringe is in line with the "ascending aorta," and so in the line in which we are recording the forces. In II, it is at $45^{\circ}$ from this line, in III at $90^{\circ}$. The "blood" has been divided into units, 3 of which are in the "heart."

Below, are schematic force diagrams for each position. $A=$ the vector when the "blood" in the syringe starts forward at systole, the recoil throwing the "body" feetward. B = the vector caused when "blood" in the "descending aorta" starts feetward, the recoil throwing the "body" headward. $\mathrm{C}=$ the vector caused when the "blood," originally in the heart, stops moving headward because the arch is reached and systole is over. $\mathrm{D}=$ the vector when the "blood" in the "descending aorta" stops as systole is over. $E=$ vectors which arise when the syringe is at an angle with the "aorta," due to acceleration of each unit of "blood" as it turns into the "ascending aorta." No vectors are given for "blood" in the "ascending aorta" because the effect of acceleration within the vessel is partly neutralized by the changes in direction when the arch is reached, and the forces resulting would be small.

The dotted line shows the system's approximate ballistocardiogram.

A schema of this kind gives only the grossest approximation of the forces involved, but it has proved more useful in giving the average reader the main features of ballistocardiographic theory than has the more elaborate and correct mathematical treatment (9). also in accord with our theory, for the change due to respiratory shifting of the heart's axis is too small to be detected in the face of the much larger changes due to the effect of the varying intrathoracic pressure on cardiac filling, and so on cardiac output.

The mask pressures we record must differ somewhat from intrapulmonary pressures when the air is moving, and they also differ from intrathoracic pressures by a factor which represents the elasticity of the stretched lungs. The latter varies with the degree to which the lungs are expanded, and this is the proper explanation for the findings which puzzled us at first: when the breath is held at full inspiration with the glottis open, the ballistic complexes remain large, and when held at full expiration they remain small until respiration is resumed. Although intrapulmonary pressure becomes atmospheric whenever the breath is held, the more the lungs are stretched, the less the intrathoracic pressure, and the greater the filling pressure, and so the output, of the heart. Therefore, large impacts follow the arrest of respiration with the lungs expanded, and smaller ones follow its arrest after expiration, as long as these positions are held.

We may now consider possible explanations for a fact seen repeatedly in the records of Figure 
3. As the impacts enlarge in amplitude during inspiration, suggesting increasing cardiac output, the systolic pressure and pulse pressure may simultaneously diminish, suggesting decreasing cardiac output. This apparent discrepancy is due to the fact that the ballistocardiogram records the sum of the impacts from the movement of blood coming from both sides of the heart while arterial blood pressure is affected by the output of the left heart only. The records obtained in F. B. with the ventricular aneurism permit insight into what is happening to the output of both sides of the heart during the respiratory cycle. The variation in height of the first " $J$ " wave suggests that, during normal inspiration, the output of the right heart increases, and during obstructed breathing, this increase is more marked, findings consistent with the old view that the greater pressure gradient during inspiration increases the filling of the right heart. The variations in height of the second "J" wave suggest that this inspiratory effect on the right heart is not immediately communicated to the left. Only after an interval of several seconds is the inspiratory increment of the right heart's output followed by a corresponding increase in left heart output, a delay due in part to the time necessary for the wave of increased pressure to traverse the lungs. Also, there is evidence that the expanding lungs have an increasing blood capacity (10) so that during inspiration more blood would run into them than runs out, a factor which would further delay the effect of the increased output of the right heart on the filling, and so on the output, of the left heart. Our data therefore, provide evidence of a new kind for a familiar physiological conception $(11,12)$ which has recently had support from the results of animal experiment (7). This conception explains the apparent discrepancy between the respiratory changes of the ballistocardiogram and arterial blood pressure.

When the lungs are subjected to violent pressure changes induced by artificial respiration with an air blast, marked effects on the circulation are seen, and these are also consistent with the conceptions given above. If sufficient pressure is used, the impacts become smaller throughout the respiratory cycle, doubtless due to the increased difficulty of cardiac filling. The small impacts reflect the reduction in cardiac output found in animals (13) and man (14) during artificial respiration of this type.

When pressure is suddenly increased, as in records 2, 3, and 5, of Figure 1, the size of the impacts does not immediately diminish although one would suppose that filling of the right heart would be diminished at once. That the latter is indeed the case is indicated by the results obtained on F. B. (Figure 1, record 7) when the first " $J$ " wave diminishes markedly as soon as pulmonary pressure is raised. The failure of the impacts to diminish as soon as this pressure is raised, is to be attributed therefore, to an increased output from the left heart. The increased height of the second " $\mathrm{J}$ " wave in F. B. (Figure 1 , record 7 ) and the rising pulse pressure after pressure in the lung has been elevated (Figure 3 , records 5 and 6 ) are in accord with this view. Doubtless blood squeezed out of the lungs by the high pressure within the alveoli maintains or even increases the left heart output for a few beats despite the immediate decrease of the output of the right heart when pressure is applied. But, the pulmonary reservoir is soon exhausted and the size of the impacts is maintained for only a few beats after such elevation of intrapulmonary pressure, and then marked diminution is always seen unless the full development of the process is interrupted by release of the pressure. Because of this mechanism, if the respiratory cycles are short, the diminution of impacts during the blast of air will not be seen, an explanation of the fact that some of these records look not unlike those secured during normal breathing.

When positive pressure is suddenly released, the effects occur in the reverse order. The increased output of the right heart is not immediately communicated to the left, not only because of the time required for the wave of increased pressure to cross the lungs, but also because blood squeezed out of the lungs by the antecedent high pressure will be replaced before left ventricular filling increases. Therefore, the full effect is not seen for some beats, and the impacts increase slowly after the pressure falls as the delayed effect on the left heart is added to the immediate effect on the right. Indeed, such delayed effects may well explain the fact that the variation in the size 
of the impacts during the normal respiratory cycle is not always a smooth curve; two maxima and two minima are seen not infrequently.

The conception that the sudden rise in intrapulmonary pressure affects the two sides of the heart differently has further support in our data. In Figure 1, record 3, the first two complexes after the sudden pressure increase are abnormal in form, the " $J$ " peak being split. While this abnormality is not as marked as in the records of F. B., the patient with ventricular aneurism, it is also to be interpreted as a dissociation of the impacts from the two sides of the heart. Apparently, filling of the right side may be so reduced by a sudden increase in pulmonary pressure that a feeble contraction follows. We have seen this abnormality only occasionally, and it has never persisted for more than a few beats. But the fact that it can be produced in persons with perfectly normal hearts by means which affect cardiac filling is of no little interest to students of ballistocardiographic interpretation.

One other point should be mentioned. According to our theoretical conceptions (9), the movement of blood caused by the contraction of the left heart makes a larger contribution to the recorded impacts than that of the right heart. Also, the results obtained in F. B., and the behavior of the blood pressure suggest that during inspiration the output of the left heart may decline, although the size of the impacts is increasing. To account for this combination of events, the respiratory changes in output of the right heart must be thought of as far larger than those of the left heart. This conclusion accords with that drawn by others (7) from measurements made on the ventricular surfaces of dogs.

Our data are, therefore, consistent with, and produce a new kind of evidence in favor of, familiar ideas concerning the effect of respiratory movements on the circulation.

On sinus arrhythmia. Our data on this subject differ from those obtained in the many animal experiments (15) and from the experience of clinicians because the ballistocardiogram not only records the rate of the heart, but also gives an indication of its output per beat. Therefore, we have analyzed our data from this point of view.

Typical records are reproduced in Figures 1 and 3. Gross inspection shows that, in general, it is the small complexes which are delivered at the slower rate, the large ones at the more rapid rate. Careful measurement shows a common exception to this generalization which is well shown in Figure 1, record 1. In this record, slowing begins just after the beginning of expiration, but the complexes maintain their height for several beats before diminishing in amplitude.

It is also of interest that, as seen in Figure 1, record 5 , the older subject, who had no sinus arrhythmia under ordinary circumstances, developed a respiratory arrhythmia when, due to diminished cardiac filling during artificial respiration with intermittent blasts of air, the size of his impacts was greatly reduced. Again, it was the smallest complexes that showed the slowing.

Another point of importance is illustrated in the record shown in Figure 1, record 3, and it has been seen repeatedly in other records. When, under artificial respiration with blasts of air, the usual relation between the size of the impacts and the respiratory cycle is reversed, the position of the slowing is reversed also. Under such conditions, the slowing occurs during inspiration, instead of during expiration. Thus, by appropriate experiments, the slowing may be driven to a different part of the respiratory cycle, but it still occurs when the complexes are small, or just before they diminish in size to give way to acceleration as soon as the complexes begin to increase in amplitude.

These results are consistent with those obtained in animal experiments as far as the rate is concerned. The observation that inflation of the lungs by a powerful blast of air causes slowing of the heart dates from 1860 (16) and has often been repeated since $(15,17,18)$, but our results permit us to make an additional statement about sinus arrhythmia.

The slowing of sinus arrhythmia in healthy persons occurs during that part of the respiratory cycle in which the cardiac output is smallest, no matter whether this occurs during inspiration or during expiration. We believe, therefore, that the arrhythmia is due to changes in the filling of the heart, rather than to the position or stretch of the lungs. The heart behaves as if it were waiting to be well filled before contracting, a mechanism 
which may be successful in maintaining stroke volume for a few beats as the rate of filling diminishes, but not for longer. Thus, these data support the well-known view $(11,12)$ that the common sinus arrhythmia of young persons is due to changes in cardiac filling induced by the respiratory movements.

\section{SUMMARY}

The genesis of the respiratory variation of the ballistocardiograms has been studied in several types of experiments.

Placing the subject on the table so that the axis of his heart in expiration was in the line in which we were recording failed to reverse the normal respiratory variation.

In a patient in the decerebrate condition, and also in normal persons, artificial respiration with an air blast did reverse the normal respiratory variation. Hence, this variation is due to changes of cardiac filling and not to changes in the position of the heart.

Records obtained on a patient with a cardiac aneurism permitted a qualitative estimate of the changes in output of each side of the heart during the respiratory cycle. Further information of this kind was obtained by simultaneous records of blood pressure and ballistocardiograms in a series of healthy persons.

The results support a familiar physiological conception as explanation for the respiratory variation of the ballistocardiogram. On inspiration, the filling, and so the output of the right heart, increases immediately, but the left heart's output does not increase until an interval of several seconds has elapsed. On expiration, the right heart output diminishes immediately, to be followed after an interval by similar diminution of the left heart's output. The change in the right heart's output during the respiratory cycle is larger than that of the left.

In the common respiratory arrhythmia of young adults, sinus arrhythmia, the slowing always occurs during expiration. Under artificial respiration with blasts of air, the slowing may occur during inspiration, but in all our records the slowing occurs when the ballistic impacts are either small or about to diminish. Therefore, our evidence supports the well-known conception that this arrhythmia is due to changes in cardiac filling $(11,12)$. It cannot be attributed to reflexes from the distension of the lungs or to phasic discharges from the respiratory center. In the sinus arrhythmia found in man, the heart behaves as if it were waiting for the right side to be properly filled before contracting.

\section{BIBLIOGRAPHY}

1. Starr, I., Rawson, A. J., Schroeder, H. A., and Joseph, N. R., Studies on the estimation of cardiac output in man, and of abnormalities in cardiac function, from the heart's recoil and the blood's impacts; the ballistocardiogram. Am. J. Physiol., 1939, 127, 1.

2. Boyd, T. E., and Patras, M. C., Variations in filling and output of the ventricles with the phases of respiration. Am. J. Physiol., 1941, 134, 74.

3. Bolton, B., Carmichael, E. A., and Stürup, G., Vasoconstriction following deep inspiration. J. Physiol., 1936, 86, 83.

4. Burton, A. C., The range and variability of the blood flow in the human fingers and the vasomotor regulation of body temperature. Am. J. Physiol., 1939, 127, 437.

5. Burch, G. E., Cohn, A. E., and Neumann, C., A study by quantitative methods of the spontaneous variations in volume of the finger tip, toe tip, and postero-superior portion of the pinna of resting normal white adults. Am. J. Physiol., 1942, 136, 433.

6. Battro, A., Segura, R. G., Eliçabe, C. A., and Araya, $E$., Influence of respiration on blood pressure in man. Arch. Int. Med., 1944, 73, 29.

7. Shuler, R. H., Ensor, C., Gunning, R. E., Moss, W. G., and Johnson, V., The differential effects of respiration on the left and right ventricles. Am. J. Physiol., 1942, 137, 620.

8. Hamilton, W. F., Brewer, G., and Brotman, I., Pressure pulse contours in the intact animal. I. Analytical description of a new high frequency hypodermic manometer with illustrative curves of simultaneous arterial and intracardiac pressures. Am. J. Physiol., 1934, 107, 427.

9. Starr, I., and Rawson, A. J., The vertical ballistocardiograph; experiments on the changes in the circulation on arising; with a further study of ballistic theory. Am. J. Physiol., 1941, 134, 403.

10. Dupee, C., and Johnson, V., Respiratory changes in pulmonary vascular capacity. Am. J. Physiol., 1943, 139, 95.

11. Bazett, H. C., In MacLeod's Physiology in Modern Medicine. C. V. Mosby Co., St. Louis, 1941, 9th Edition.

12. Starling's Principles of Human Physiology. J. and A. Churchill, Ltd., London, 1936, 7th Edition. 
13. Humphreys, C. H., Moore, R. L., Maier, H. C., and Apgar, V., Studies of the cardiac output of anesthetized dogs during continuous and intermittent inflation of the lungs. J. Thoracic Surg., 1938, 7, 438.

14. Cournand, A., Personal communication.

15. Anrep, G. V., Pascual, W., and Rössler, R., Respiratory variations of the heart rate. $I$. The reflex mechanism of the respiratory arrhythmia. Proc. Roy. Soc., B., 1936, 119, 191.
16. Einbrodt, M., S. B. Akad. Wiss. Wien. 1860, 40, 345 cited from Anrep et al. (15).

17. von Saalfeld, E., Herzreflexe pulmonalen ursprungs. Arch. f. de ges. Physiologie, 1932, 231, 33.

18. von Saalfeld, E., Venöser Blutdruck und Herzreflexe pulmonalen ursprungs. Arch. f. de ges. Physiologie, 1932, 231, 724.

19. Nickersen, J. L., and Curtis, H. J., The design of the ballistocardiograph. Am. J. Physiol., 1944, $144,1$. 